\title{
A COMPREHENSIVE APPROACH INTO STEREOSCOPIC VISION
}

\author{
Lely Retno Wulandari ${ }^{1}$ \\ Correspondence:lely_wulandari@ub.ac.id \\ ${ }^{I}$ Ophthalmology Department, Faculty of Medicine, Universitas Brawijaya, Malang, Indonesia
}

\begin{abstract}
Article History:
Received: December 12, 2021

Accepted: December 27, 2021

Published: January 1, 2022

\section{Cite this as:}

Wulandari LR. A comprehensive approach into stereoscopic vision. Malang Neurology Journal; 2022.8:53-.57. DOI: http://dx.doi.org/10.21776/ub.mnj .2022.008.01.11
\end{abstract}

\begin{abstract}
Stereopsis (or stereoscopic) vision is the ability to see depth of perception, which is created by the difference in angle of view between both eyes. The first process is known as simultaneous perception. Objects will fall on each corresponding retina and there will be a process of fusion of the two images into one. Then, the brain initiates three-dimensional perception in visual cortex, creating stereoscopic vision. Stereoscopic vision will rapidly develop, especially at the age of 6-8 months of life. Stereoscopic is important in daily activities. There are many stereoacuity tests to evaluate stereoscopic vision. Stereoscopic examinations are based on the principle of haploscope, anaglyph, or polaroid vectograph. There are qualitative and quantitative examination methods to assess stereoscopic vision. Qualitative examinations such as Horizontal Lang Two Pencil test and Synoptophore. Quantitative examination including Contour stereopsis test and Clinical random dot stereopsis test. The inability of the eye to see stereoscopic can be called stereoblindness. This can be affected by amblyopia, decreased visual acuity, or the presence of ocular misalignment. Inability to achieve stereoscopic vision will impact an individual to perform some daily life activities, and lead to an increase in difficulty interacting in the world.
\end{abstract}

Keywords: Stereopsis, stereoacuity test, stereoblindness

\section{Introduction}

Stereopsis (or stereoscopic) vision is the capability to recognize depth, created from the difference in perspectives of both eyes. Functionally speaking, stereopsis is essential for humans to accurately estimate distances, recognize, understand, and place objects, and perform activities involving smooth hand movements. Stereopsis provide a sense of volume (the space between objects) in the visual world. ${ }^{1}$

Stereopsis is derived from the Greek word stereo, which means "dense", and opsis, which means "appearance", "vision". Stereopsis is a term oftentimes used in elaborating the perception of depth and three-dimensional structures obtained from visual information. This information is required to come from two individual eyes that have perfectly developed binocular vision. ${ }^{2,3}$

External area is projected into both eyes' retina as twodimensional images which is slightly displaced from one another (retinal disparity). Brain fuses these two images, aiming to attain relative and absolute depths of objects. This facility for binocular depth perception assists in shape-recognition of objects and it also important for viewing objects in motion, because it permits an assessment of trajectories through space. ${ }^{4}$

\section{Mechanism of Stereoscopic Vision}

The reason why stereopsis takes place is the object that we see falls on both of our retinas, which have a small disparity due to the horizontal distance between the left eye and the right eye, thus integrating cortically and forming the perception of depth (Figure 1). ${ }^{5}$

After both eyes can see the image separately, the brain then combines these produced images. As an example, if the right eye sees the upper half part of a pine tree and the left eye sees the bottom half, the brain must be able to incorporate the two images to see a complete image of a whole pine tree. This process is called the fusion process. First, both eyes must be able to see one object at the same time, then the images are then merged. This second process is what we call the fusion process. The next process is the analysis process. The brain then analyzes the objects in an image in one eye and compares them to that of the other. By observing the subtle differences in the two produced images by virtue of the distance between our eyes, the brain creates a 3D perception in visual cortex. This process is called stereopsis. ${ }^{3}$

Many researchers observe various visual area that responsible for binocular vision. Neurons that specifically respond to binocular disparity were first described three decades ago. Until now, area that are working for binocular disparity vision including MT, MST, V1, V2, and V3. Even though disparity-selective neurons are thought to form the neural substrate for stereopsis, the mere existence of disparity-selective neurons does not assure that they make contributions to stereoscopic depth perception. Some disparity selective neurons may additionally play other roles, including guiding vergence eye movements. 7 


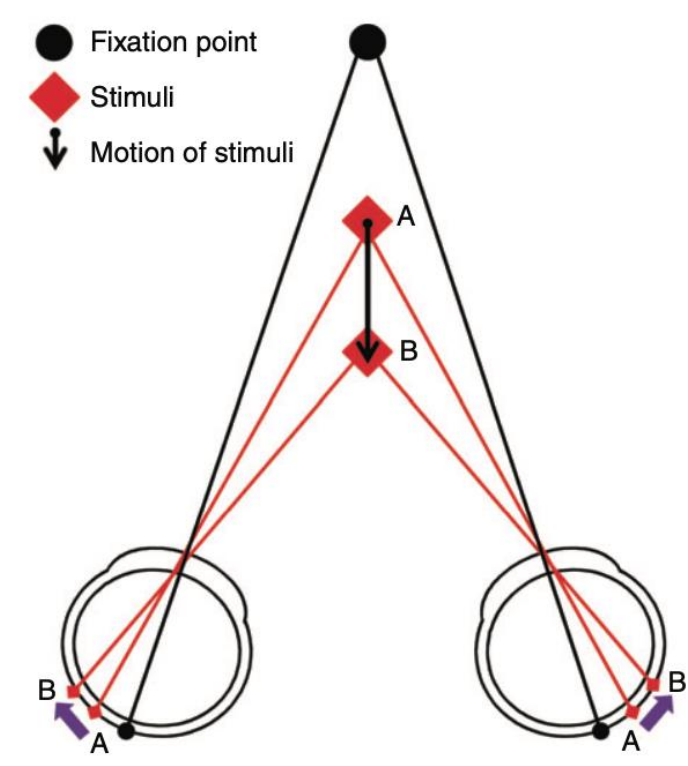

Figure 1. Distance between two eyes cause retinal disparity.

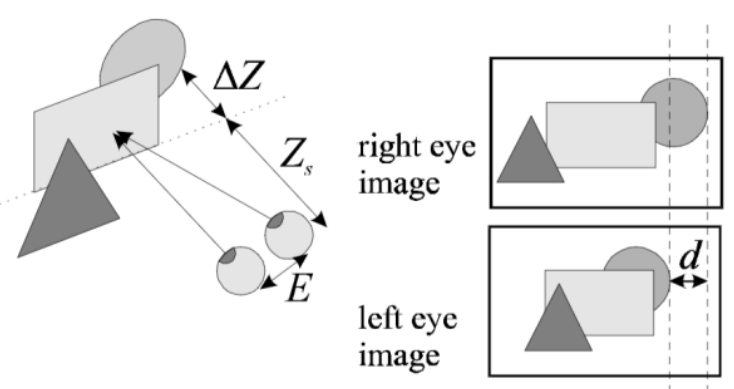

Figure 2. The process of simultaneous perception

Depth perception is not innate. Stereopsis is haven't been achieved in infants less than 3 months old. Past this age, stereopsis develops rapidly, particularly at the age of 6-8 months. Interestingly, this development process exceeds the rate at which visual acuity matures. ${ }^{8}$

The steps of stereopsis are gradually attained from visual information. Newborns take up their first 8 years of life to perfect their stereopsis ability. As previously mentioned, the critical period of the stereoscopy formation process in humans begins at the age of 3 months, followed by a swift maturation period at the age of 6,8 , until 18 months and progressive improvements until the age of 3 years. Recent research suggests that the process of stereopsis formation remains evolving after the age of 3 years and overlaps with the development process of visual acuity. ${ }^{9}$

There are morphological changes including eye growth, corneal diameter increase, and connection formation between eyes and the associated cortex that continuously develops as newborns age. The functions of neurons in the cortical and subcortical structures improve progressively. The developments of visual perception with age include visual acuity, color perception, contrast sensitivity, binocular vision, and 3D perception, all of which experience improvements. The entire process takes place during a "critical period" of the development. Should there be any abnormalities, such as strabismus, they will cause amblyopia and the loss of binocular vision. ${ }^{10}$

\section{The Principles Of Stereopsis Examinations}

\section{How to examine stereopsis?}

Each individual's stereoscopic ability differs from another. The quantification of stereoscopic visual acuity is called stereoacuity. The assessment of stereoacuity in clinics employs second of arc or arcsecond. One arcsecond is equal to $1 / 3600$ of a degree. The obtained results from stereoacuity heavily depend on the type of assessment used for stereoscopic examination, and the normal range lies between $30-40$ arcsecond. $^{11}$

Stereopsis is measured in arcseconds. When looking at an object, the right eye and the left eye have a different line of sight. The line of sight of the right eye and the left eye converges on a fixation point. It is at this point that the two lines form an angle that we call visual angle. The angle formed upon seeing an object closer to us will be wider. Thus, the difference in the two angles will provide a relative distance between two objects. The two angles are expressed in arcseconds in stereopsis. This states how much arcminute is acceptable. When the angles are infinitesimal, it can be concluded that the two objects are close to each other. The stereoscopic vision of an individual is quantitatively stated as the smallest arcsecond of depth perceivable binocularly. The closer the object to the eye, the better the stereopsis. ${ }^{12}$

\section{Haploscope, anaglyph \& polaroid vectograph}

All stereoscopic examinations are based on one of the three principles. Technically, a haploscope is an optic device used to provide different images simultaneously for each eye. A pair of mirrors is placed 45 degrees from each other. The right mirror will display an image for the right side and the left one for the left side. Using a haploscope, we can see both images at the same time. Due to the similarities in the images, the brain is able to incorporate and process them. ${ }^{13}$

The principle of the Anaglyph technique uses different colors to present different images. This technique requires a pair of glasses with a red filter on the right and a green filter on the left. Through this apparatus, the right eye will only see a set of red-colored pictures and the left one will only see a set of green-colored images. The TNO test for stereopsis is based on anaglyph. ${ }^{13}$

Lastly, the principle of the Vectograph is centered on the fact that light waves can be polarized. A light ray traveling in a given room has consist of many waves. There can be some horizontal waves, some vertical waves, and some others on a particular angle. The mechanism underlying this examination is that the waves are stopped by a barrier with thin vertical slits, hence only allowing the verticallyoscillating waves to pass through and the rest are blocked. In a vectograph, there will be a pair of different images covered with a polarizing film. An individual undergoing this examination will wear a set of polarized glasses in which the polarizing angles differ from each other. The right eye and the left eye will only see the verticallyoscillating light wave and the horizontally-oscillating wave, respectively. The vectograph technique governs the Titmus test. The test image consists of a fly with different depths printed on various parts of the large fly image. The flies can be replaced with shapes or other quotidian images. ${ }^{13}$ 


\section{Qualitative Techniques for stereoscopic examination}

Horizontal Lang Two Pencil Test, a simple test with a high sensitivity. This test can be use as a screening tool to find out global stereopsis and binocularity. The examiner holds a pen vertically, and sit in front of the patient. The patient also holds a pen vertically. The patient's need to bring the two ends of the pen together. The patient mostly able bring together the two ends with both eyes open. The patient will be fails when one eye closes or when both eyes are open but there is no stereoscopic vision, this process described in figure $3 .^{14}$

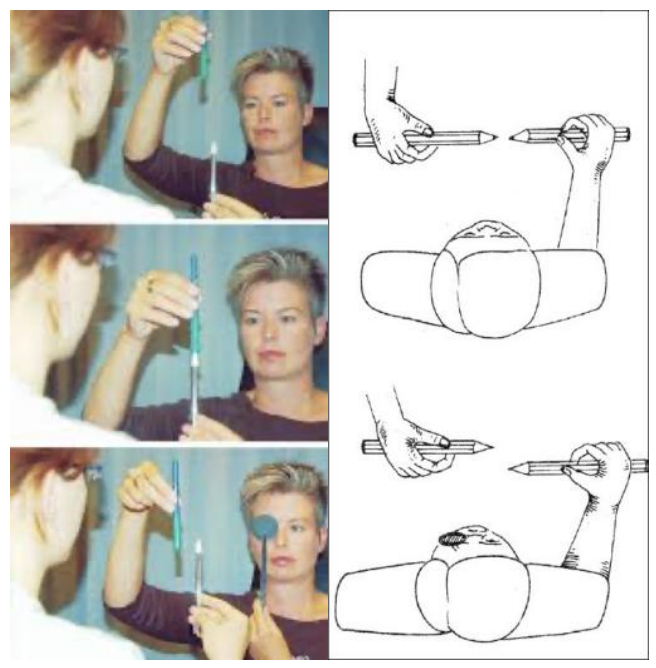

Figure 3. The Horizontal Lang two-pencil test. The patient's need to bring the two ends of the pen together.

Synoptophore, also known as major amblyoscope, is a multipurpose instrument that can be used to subjectively assess ocular motility. For the subjective measurement, a different target will be visible on each eye, and the patient is asked to place them in a superimposed position. In a normal retinal correspondence, the examination result will show that the horizontal, vertical, and torsional deviation can be immediately observed from the major amblyoscope scale. Synoptophore can be used for examining objective and subjective deviation angle, normal and abnormal retinal correspondence, cyclophoria, hyperphoria, and horizontal and vertical vergence. This assessment is able to test the three binocular vision levels, which are simultaneous perception, fusion, and stereopsis. Qualitative stereopsis assessment can be obtained using two images, taken from a slightly different angle in order to illustrate depth perception. (Figure 4)

\section{Quantitative Techniques for stereoscopic examination}

\section{Contour stereopsis test and Clinical random dot stereopsis test}

The contour stereopsis test widely used is the Titmus stereopsis test. The book placed $40 \mathrm{~cm}$ in front of patient, with the patient wearing polarized glasses. The examination booklet is perpendicular to the subject's visual axis. The fly is shown first, and if a positive response is given, the subject is then asked to identify the circle and a different animal in each set, including the fly $(3,600$ arcsec $)$, the animal (100 arcsec to 400 arcsec), and Wirt cycle (800 $\operatorname{arcsec}$ to $40 \operatorname{arcsec})$. The examination technique comprises holding a straight image in front of the patient to maintain a proper polarization axis. Ensure good lighting but avoid glaring surface reflection. . $^{5,15}$
The ability to recognize the position of the fly's wings on a plane of cards suggests crude stereopsis (3000 arcseconds). A better stereoacuity level can be shown by using stereoscopic images with a smaller horizontal disparity; in each examination, the patient must identify one image provided stereoscopically from a group of similar images. ${ }^{5}$
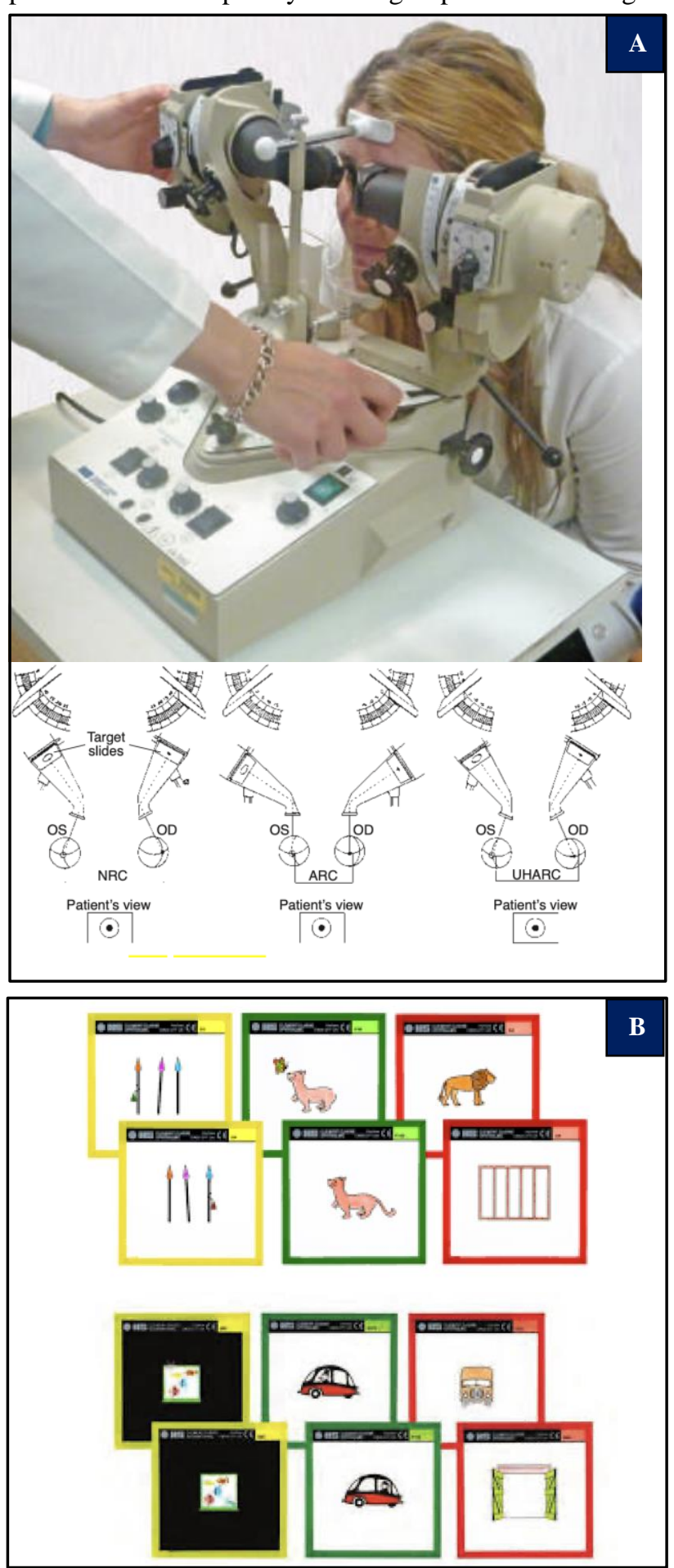

Figure 4. Synoptophore, the major amblyoscope. (A) The target can be placed on each of the instrument's arms to provide for each eye. The arm of the instrument can be shifted to observe the magnitude of eyeball motility. (B) The slide in red for the simultaneous perception process, the slide in green for the fusion process, and the slide in yellow for the stereopsis process. Each slide place on each arm of synoptophore. 


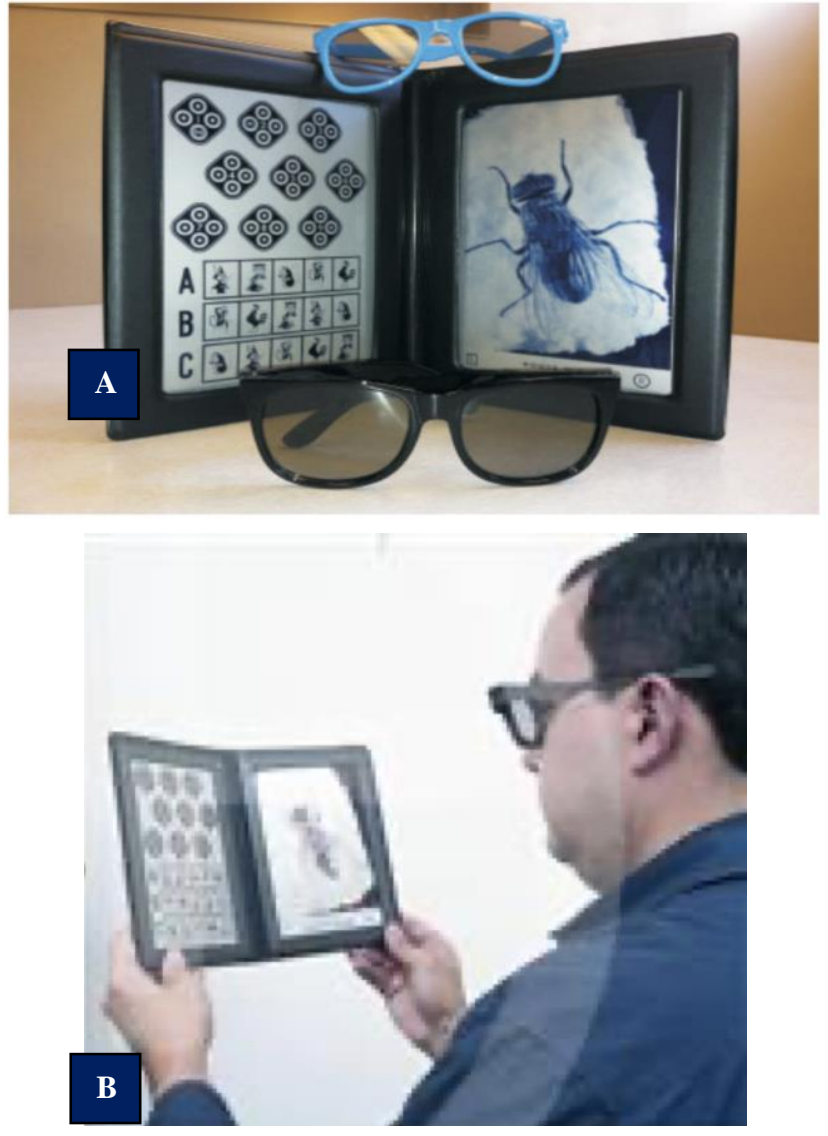

Figure 5. (A) The Titmus Test, tested using polarized glasses . Patient needs to recognize the fly's wing and one image provided stereoscopically from a group of similar images. (B) The position of patient while doing Titmus test by using polarized glasses.

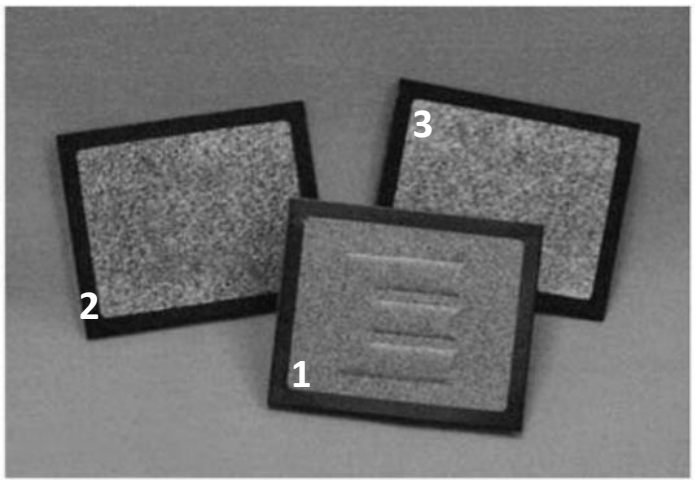

Figure 6. Stereotes RDE comprises three stereoscopic test plates: (1) a stereo E test plate, it contain random dots that will seen as an E letter, when viewed binocularly through polarized glasses; (2) a model $\mathrm{E}$ test plate containing a raised, three-dimensional E; and

(3) a blank card containing random dots but no stereo target.

\section{Clinical Random Dot Stereopsis}

Clinical Random Dot Stereopsis consists of Random Dot E (RDE.), TNO (Lameris Ootech), Frisby and Lang stereotest. Randot Stereotest is made of several parts, some of them have the random dot stereopsis format and the others with monocular contour. In selecting a stereopsis test, there are a number of important things to consider. First, a test must contain no or few monocular cues. Stereotest Random Dot E (RDE) fulfills this prerequisite and has been recommended to be used in preschool visual screening. (Figure 6). ${ }^{16}$

TNO stereo test (Nederlandse Organisatie voor Toegepast Natuurwetenschappelijk Onderzoek) is a common test used to evaluate stereoscopic vision where patient need to using anaglyph glasses. As tradtional method for evaluating stereopsis, TNO is also a stereotest used for more than 40 years. This test is both uncomplicated to perform and it is also easy for the evaluated patient to understand. ${ }^{17}$

\section{The Importance of Stereopsis}

The concept of stereoscopic vision makes us realize the importance of our both eyes. Generally, the horizontal separation between both eyes allows them to see the same object from two different lines of sight. Stereopsis can be achieved should the following be fulfilled: normal eyeball position (orthophoria) and good visual acuity of each eye.

Stereoscopic vision can only be achieved with the presence of a simultaneous and superimposed perception process. The two objects will fall in each corresponding retinas and the fusion process then takes place. Because stereoscopic vision relies on a good vision of both eyes, adequate oculomotor control, and the development of binocular brain mechanisms, stereopsis is frequently considered the highest degree of binocular visual function. ${ }^{9}$

Stereopsis makes it possible for us to perform various activities with ease, such as driving and parking a car, pouring a cup of coffee, inserting needles, stitching and sewing, ascending and descending stairs, reaching, touching, holding, and performing surgeries. ${ }^{9}$

Possessing stereoscopic vision is a goal that should be attained. Perfect stereopsis will help precise and accurate hand movements. Both children and adults with stereoscopic dysfunction will perform poorly in multiple visuomotor activities in comparison to their peers with a normal stereoacuity. Good stereoacuity is also correlated with better reading ability. Some daily activities require perfect vision on both eyes and a reasonable degree of oculomotor coordination. ${ }^{9}$

\section{Stereoscopic vision-related disorders}

The total absence of stereoscopic vision is called stereoblindness and partial dysfunction has several names: stereoimpairment, stereoanomaly, or stereodeficiency. Stereoblindness is the inability to perceive $3 \mathrm{D}$ shapes using stereo vision, which renders them incapable of sensing stereoscopic depth by combining and comparing an object from both eyes. ${ }^{1}$

The prevalence of stereoblindness in the general population starts from $1 \%$ to $64 \%$. Adrien Chopin (2019) estimated the prevalence of stereoblindness to be $7 \%{ }^{1}$

In order for the binocular visual function to take place, newborns and infants must coordinate both of their eyeball motility through the integration of sensory information nerves from their eyes to achieve the binocular vision in a dynamic, three-dimensional environment. This coordination seems to be inhibited when they suffer from strabismus in the first couple of years after their birth.

Banks et al have demonstrated the importance of uniform binocular visual experience throughout the first years of their life. The cause of stereoblindness in strabismus is the central process, at least in several cases. As elaborated above, each abnormality in the visual pathway (due to abnormal retinal wave before birth or others) can result in abnormal visual perception as a result of central processes. 
So, for instance, unusual segregation of the inputs from both eyes and/or other problems on the retina will cause an atypical ocular dominance map development and/or abnormal retinotopic map in the visual cortex. This will lead into abnormal binocular integration. Abnormalities in the orientation and/or spatial frequency mapping in V1 (or more) can also give rise to amblyopia, and loss of binocular vision. More commonly, every change in the paths $\mathrm{M}, \mathrm{P}$, or $\mathrm{K}$ from the retina to the cortex, will lead to such changes. ${ }^{7}$

Abnormal development of stereopsis may be due to amblyopia, reduced visual acuity as a result of suppression scotoma, uncorrected refractive errors, and visual stimulus deprivation. Amblyopia is decrease visual function, caused by abnormality throughout growth. Visual deprivation amblyopia occurs in patients with constant obstruction in the mechanism of retinal image formation, such as congenital ptosis, traumatic or congenital cataract, and prolonged corneal clouding left untreated for a long period of time. ${ }^{15}$

Stereoblindness and stereoimpairment may arise from different causes. Holmes \& Horax described that a patient with parietal cortex damage will result in stereoscopic disturbance. Moreover, stereopsis is often impeded in patients with age-related macular degeneration (AMD). Reduced stereoacuity is also influenced by age. Stereoacuity decreases in patients with cataract and glaucoma. ${ }^{1}$

\section{Conclusion}

Stereopsis is really important in humans for accurate object recognition and object understanding. There are several tests that can be used to assess stereoacuity, either qualitatively or quantitatively. A person's inability to see stereopsis can really affect daily activities and quality of life.

\section{Conflict of Interest}

The Authors declare that there conflict of interest.

\section{Acknowledgement}

None

\section{References}

1. Chopin A, Bavelier D, Levi DM. The prevalence and diagnosis of 'stereoblindness' in adults less than 60 years of age: A best evidence synthesis. Ophthalmic and Physiological Optics; 2019. Mar;39(2):66-85. DOI: 10.1111/opo.12607

2. Howard IP, Rogers BJ. Perceiving in depth, volume 2: Stereoscopic vision. Oxford University Press; 2012 Jan 27.

DOI: 10.1093/acprof:oso/9780199764150.001.0001

3. Vishwanath D. Toward a new theory of stereopsis. Psychological review; 2014. Apr;121(2):151.

DOI: $10.1037 / \mathrm{a} 0035233$

4. Freeman RD. Stereoscopic vision: Which parts of the brain are involved? Current biology; 1999. Aug 26;9(16):R610-3. DOI: $10.1016 /$ S09609822(99)80386-8

5. O'connor AR, Tidbury LP. Stereopsis: Are we assessing it in enough depth? Clinical and
Experimental Optometry; 2018. Jul 1;101(4):485-94. DOI: $10.1111 /$ cxo. 12655

6. Lagendijk RL, Franich RE, Hendriks EA. Stereoscopic image processing. Delft University of Technology, Electrical Engineering, Delft, The Netherlands; 2002.

7. DeAngelis GC, Cumming BG, Newsome WT. Cortical area MT and the perception of stereoscopic depth. Nature; 1998. Aug;394(6694):677-80.

DOI: $10.1038 / 29299$

8. Von Noorden GK. Binocular vision and ocular motility. Theory and management of strabismus; 1990. ISBN: 0-323-01129-2

9. Fawcett SL, Wang YZ, Birch EE. The critical period for susceptibility of human stereopsis. Investigative ophthalmology \& visual science; 2005. Feb 1;46(2):521-5. DOI: 10.1167/iovs.04-0175

10. Bui Quoc E, Milleret C. Origins of strabismus and loss of binocular vision. Frontiers in integrative neuroscience; 2014. Sep 25; 8:71.

DOI: 10.3389/fnint.2014.00071

11. Deepa BM, Valarmathi A, Benita S. Assessment of stereo acuity levels using random dot stereo acuity chart in college students. Journal of family medicine and primary care; 2019. Dec;8(12):3850.

DOI: $10.4103 / j f m p c . j f m p c \_755 \_19$

12. Read JC. Stereo vision and strabismus. Eye; 2015. Feb;29(2):214-24. DOI: 10.1038/eye.2014.279

13. Piano ME, O'Connor AR. The effect of degrading binocular single vision on fine visuomotor skill task performance. Investigative ophthalmology \& visual science; 2013. Dec 1;54(13):8204-13.

DOI: $10.1167 /$ iovs.12-10934

14. Nongpiur ME, Sharma P. Horizontal Lang two-pencil test as a screening test for stereopsis and binocularity. Indian journal of ophthalmology; 2010. Jul;58(4):287. DOI: $10.4103 / 0301-4738.64125$

15. McIntire JP, Havig PR, Harrington LK, Wright ST, Watamaniuk SN, Heft EL. Clinically normal stereopsis does not ensure a performance benefit from stereoscopic 3D depth cues. 3D Research; 2014. Sep $1 ; 5(3): 20$.

16. Vision in Preschoolers Study Group. Random Dot E stereotest: Testability and reliability in 3-to 5-year-old children. Journal of American Association for Pediatric Ophthalmology and Strabismus; 2006. Dec 1;10(6):507-14. DOI: 10.1016/j.jaapos.2006.08.019

17. Zhang Y, Meng B, Wu H. Evaluating the mechanism by which the TNO stereo test overestimates stereo thresholds. Journal of Ophthalmology; 2021. Jan 18. DOI: $10.1155 / 2021 / 6665638$

18. Khazaeni L, Quinn GE, Davidson SL, Forbes BJ. Amblyopia treatment: 1998 versus 2004. Journal of Pediatric Ophthalmology \& Strabismus; 2009. 46(1):19-22. DOI: 10.3928/01913913-20090101-1 